\title{
Experiment on Winding of Roving
}

Part 2: Effects of Some Factors upon Winding of Roving.

\author{
By T. Isshi, M. Nakazawa and S. Matsuhashi, Members, TMSJ \\ Faculty of Textile Science and Technology, Shinshu University, Ueda, Nagano.
}

Based on the Journal of the Textile Machinery Society of Japan, Transactions, Vol. 20, No.6, T 162-17I (1967); Vol.20, Nos.7/8, T 205-210 (1967)

\section{Abstract}

\begin{abstract}
In the previous instalment, author Isshi suggested a method of investigating the winding of roving and studied presser pressure on a roving package as one of the factors in the winding of roving. (1) Until then, there had been hardly any published reports concerning experiments on the subject. We have carried out investigations of other factors with the results which are summed up as follows:

1) When the frictional resistance of a flyer to roving is large, the roving becomes coarse in the early stage of winding, but depending on the character of the winding mechanism, factors such as roving tension and roving weight of unit length tend to approach a constant value as layers of roving increase in number.

2) We have clarified the correlation among the spindle position, the number of false twists of a roving and the roving weight, and proved quantitatively the usefulness of "rotafil" as an aid to a flyer.

3) Fluctuations in roving weight in each layer in the early stage of winding (when layers are small in number) are presumably caused, in the main, by improper diameter of the package. These fluctuations become gradually small as layers increase in number, because the self-controlling mechanism of winding corrects the improper diameter. Fluctuations in roving weight in the closing stage (when layers are large in number) are presumably caused, in the main, by improper relative revolutions of the bobbin and the flyer.

4) With a small number of relative revolutions of the bobbin and the flyer, roving weight initially appears large but later becomes proper gradually, due to the self-operating control of the winding mechanism.

5) The winding pressure of the presser on the package is presumably one of the most important factors which make high-speed winding possible.
\end{abstract}

\section{Introduction}

As stated in the previous instalment, there are two groups of factors which show the conditions for and the state of the winding of a roving $[1]$. The first group, uninfluenced by any other factors, includes, inter alia, the relative revolutions of the bobbin and the flyer, the type of winding of a roving on the flyer and the number of spindle revolutions.

The other group consists of roving weight, the diameter and stiffness of the roving package and roving tension. Each of these factors is influenced by every first-group factor as well as by every other factor in the second-group.

The present instalment deals with five factors in the firstgroup and measures variations in the second-group factors by varying the values of the five factors in the first-group. a) Type of winding of roving on flyer

This factor is obtainable in various types by varying the type of winding of a roving on the flyer top and the number of turns of the roving around the presser paddle. From a physical point of view, these treatments amount to little more than varying the resisting force of a flyer to roving. b) Number of false twists of roving and position of spindle

It is common knowledge that roving weight in the front row of bobbin rail differs from that in the back row [2]. This difference is removable by equipping flyers with "rotafil" which are now often used $[3,4,5]$. Attaching a rotafil to a flyer gives enough false twists to a roving and, therefore, increases its resistance to elongation. 
c) Traverse motion of bobbin rail

The mechanism of the traverse motion of bobbin rail has been analyzed precisely $[6,7,8]$. However, the relation between the traverse motion and roving weight is not easy to analyze. There have hardly been any attempts made to analyze it. Yet this relation needs clarification for correct adjustment of roving weight.

d) Relative revolutions of bobbin and flyer

The relative revolutions of the bobbin and the flyer mean the revolutions of the bobbin relative to those of the flyer. They seems to be the most basic factor in the winding of roving, besides being an important factor in any effort at automatic control of the winding mechanism of the roving frame.

e) Spindle revolutions

Presser pressure is a highly influential factor in highspeed winding, but it is apt to be overlooked.

\section{Winding of Roving on Flyer}

\section{2-1. Experimental Procedures}

The roving frame used in our experiment was a Howa simplex frame (1945 make) with a 10-inch lift. The slivers used were those for $20 \mathrm{~S}$ cotton yarn. The twist of roving was fixed at 1.59 turns per inch. The diameter of the roving package was measured continuously by a silicon photo-cell fitted to the flyer leg and by a shutter placed on the presser.

Roving tension on the flyer top was measured by finding the amount of displacement of the tension cap supported by a coil spring on the flyer top. The weight of the roving was obtained by measuring the weight of a 10-yard roving at a temperature of $20^{\circ} \mathrm{C}$ and a relative humidity of $65 \%$. The stiffness of the roving package was expressed by a gram weight for compressing $2 \mathrm{~mm}$ in the diameter of layered package [1].

\section{2-2. Results of Experiment Discussed}

Two types of winding of roving on the top of a flyer were used in our experiment. One was right-hand winding, in which the roving was so placed as to go round the fiyer top in a clockwise direction. This type of winding is common in practice. The other was left-hand winding, in which the roving passed through the flyer top in the direction reverse to right-hand winding. Three numbers (1,2 and 3$)$ were used as the number of turns of the roving around the presser paddle.

Figs. $1-3$ and Table 1 give the results on the two types of winding on the flyer top. Figs. $4-6$ give typical data on the effects of the number of turns. These figures were included in the previous article but are repeated here for discussion from another point of view.

$\boldsymbol{P}_{a}$ and $\boldsymbol{P}_{b}$ in Fig. 4 express the types of flyer classified by the character of presser pressure. The sub-graphs of Fig. 4 show the characteristic curves of presser pressure, the horizontal coordinate denoting the diameter $(\mathrm{mm})$ of the roving package and the vertical coordinate denoting presser pressure $(\mathrm{g})$.

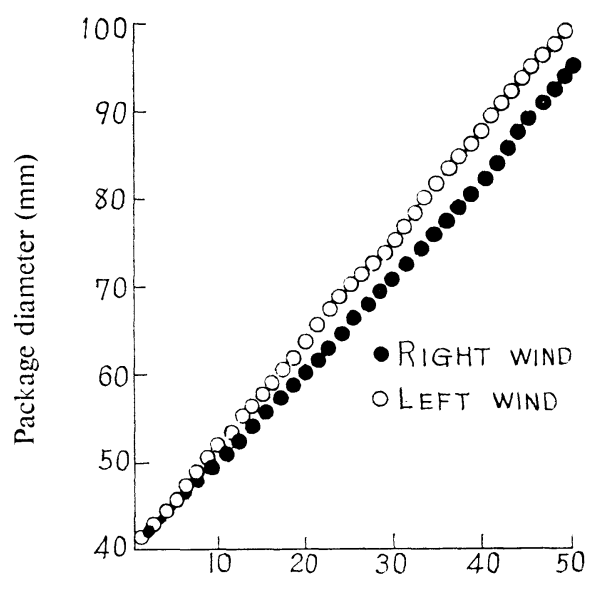

No. of layers

Fig.1 Package diameter (type of winding on flyer top)

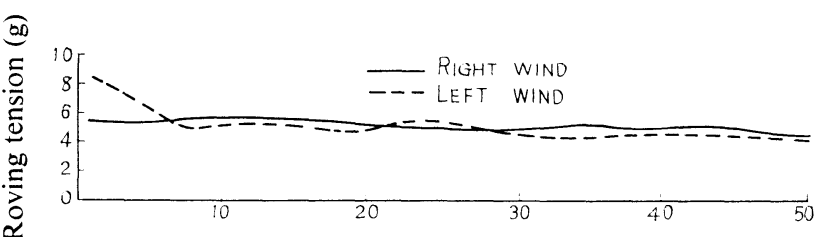

No. of layers

Fig.2 Roving tension (type of winding on flyer top)

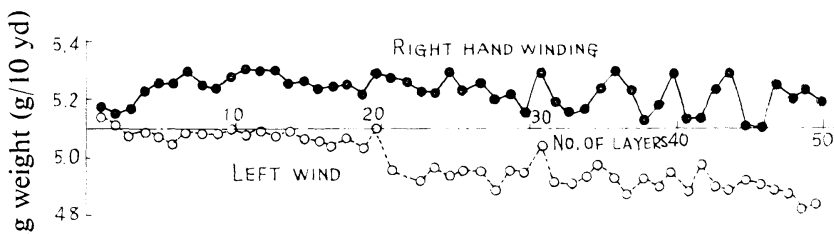

Fig.3 Roving weight (type of winding on flyer top)

Table 1 Stiffness of Package

\begin{tabular}{cc} 
Right winding & Left winding \\
\hline 410 & 275 \\
\hline
\end{tabular}

A comparison of the two types of winding on the flyer top shows that right-hand winding gives a roving more resistance force than does the left-hand winding. The larger the resisting force, the heavier the roving tension and the heavier the winding pressure of the presser. The winding pressure of the presser means the compressive force of the presser on the package. It consists of presser pressure produced by the centrifugal force of the hollow leg and the component of winding tension, i.e., the roving tension of the presser paddle on the package in a radial direction. The heavier the winding pressure of the presser, 
the denser the packoage wund and the smaller its diameter. The winding speed of the bobbin is proportional to the product of the number of relative revolutions and the package diameter. Therefore, the smaller the diameter of a package, the less the winding draft (the ratio of the winding speed of roving to the delivery speed of the front roller), with roving weight tending to increase gradually.

The experimental results given in Figs. $1-3$ and Table 1 largely bear out what we have said in the foregoing.

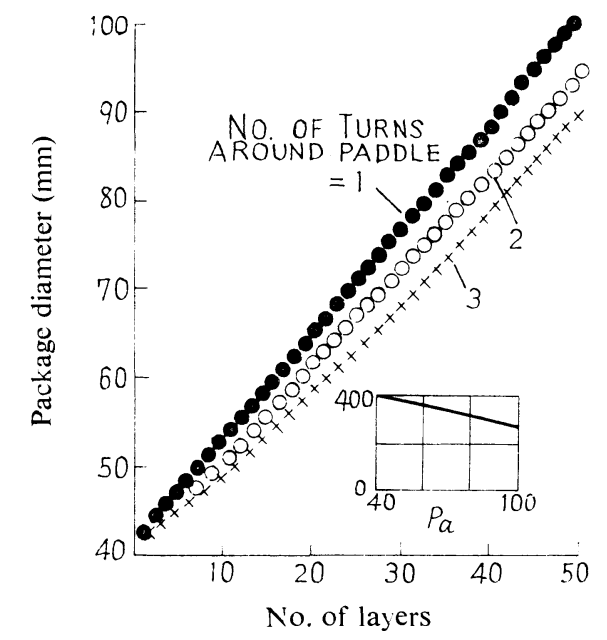

(a) Presser type $P_{b}$

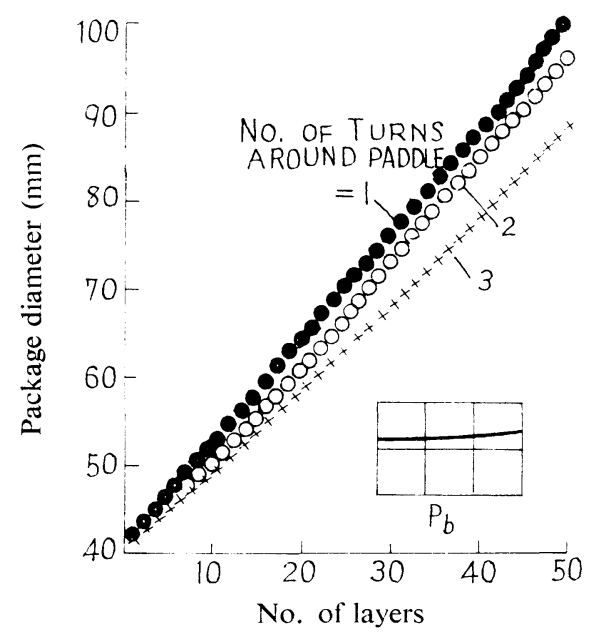

(b) Presser type $P_{b}$

Fig.4 Package diameter (type of winding on presser)

Figs. $4-6$ show the effects of the number of turns of a roving around the presser paddle and prove that the effect of one turn has the same tendency as that of left winding in Figs. 1-3; and that the effect of three turns has the same tendency as that of right-hand winding. This relation proves that varying the type of windig of roving on the flyer top and varying the number of winding of turns of the roving around the presser paddle are nearly the same in their effects on winding of a roving, and that their

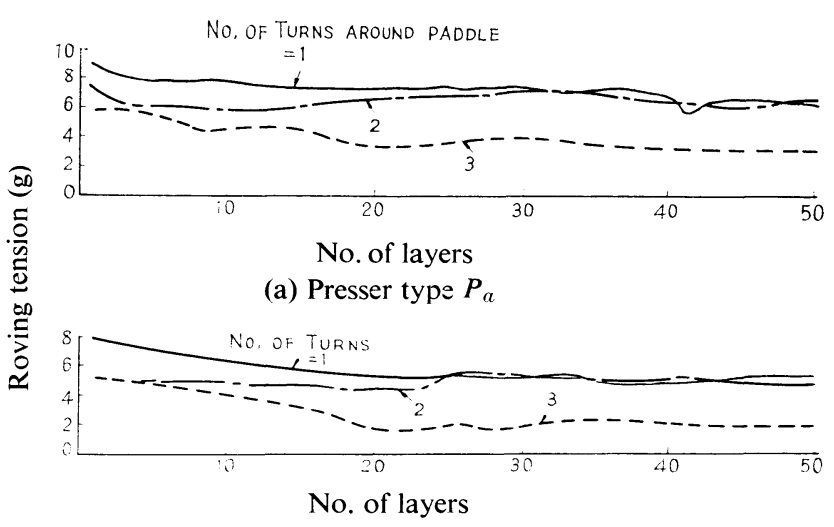

(b) Presser type $P_{b}$

Fig.5 Roving tension (type ofwinding on presser)

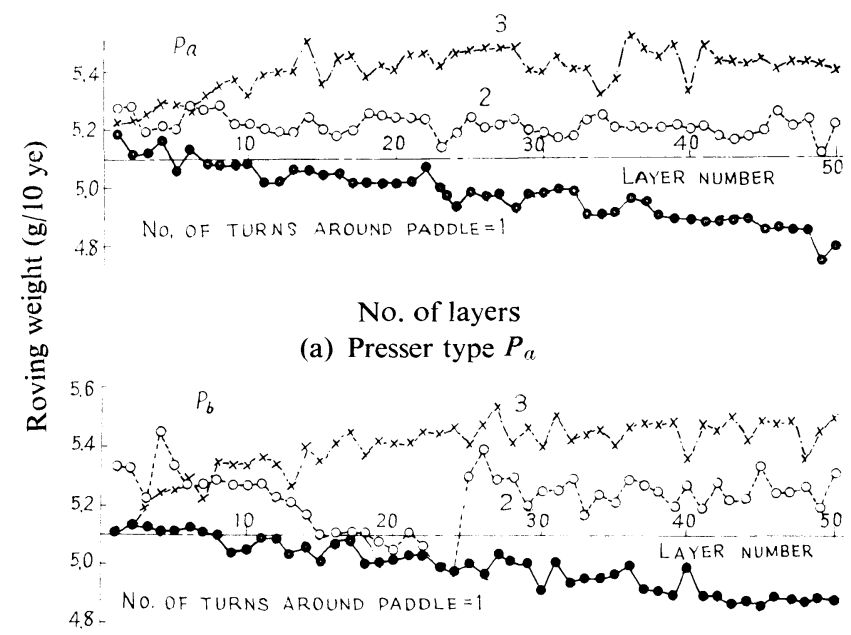

No. of layers

(b) Presser type $P_{b}$

Fig 6 Roving weight (Type of windining on presser)

effects show up in variations in the frictional resistance of the flyer to a roving.

The correlation of the factors probed may be summarized as in the following flow chart:

[Frictional resistance of flyer]
(large)
Winding tension
(large)
(increases slowly)
Winding speed
(slow)
(smanding draft
(large)
denotes the factors measured in our present experiment


This chart holds good even if every value expressed in ( ) is substituted by exactly the opposite value. It is not possible to explain, however, why fluctuations in roving tension, roving weight, etc. caused by varying the type of winding on the flyer do not increase cumulatively with the growth of the package.

Figs. 1-6 show the presence of the negative feed-back mechanism shown in the following flow chart:

$$
\begin{aligned}
& \text { Roving weight } \\
& \text { (large) } \\
& \hline \text { Package diameter } \\
& \text { (increases rapidly) } \\
& \text { Winding speed } \\
& \text { (rapid) } \\
& \text { Winding draft } \\
& \text { (large) } \\
& \text { Roving weight } \\
& \text { (small) }
\end{aligned}
$$

Let it be added that fluctuations in roving tension are not as distinct as those in the other factors; and that, therefore, we had better not take roving tension as the control value for the control of the roving frame.

\section{Number of False Twists of Roving and Position of Spindle}

\section{3-1. Experimental Procedures}

In order to give a roving more false twists, some of our tcst flyers were equipped with a rough finished top hole surface. We call these flyers "Special flyers". The number of false twists obtained from the special flyers were counted from their photograph. The other procedures were the same as described in the previous section.

\section{3-2. Results of Experiment Discussed}

The false twists in the back row exceed those in the front row in experimental value (Table 2). The effects of the difference in the spindle position are plainly shown as the difference in roving tension (Fig. 8), in roving weight (Fig. 9) and in package stiffness (Table 3).

We may say, in the light of the figures and table cited, that the differences adduced are caused only by the difference in the number of false twists of a roving. This statement is based on the fact that a comparison of the special flyers (which give more false twists) and the ordinary flyer in their effects exhibits a similar tendency between the frontrow and back-row twists. This speaks well for "rotafil."

Table 2 No. of False Twists

\begin{tabular}{ccc}
\hline & Large false twist & Ordinary false twist \\
\hline Front row & $25 / 32-20 / 32$ & $13 / 32-10 / 32$ \\
Back row & $(0.78-0.62)$ & $(0.40-0.31)$ \\
& $18 / 19-14 / 19$ & $13(19-10 / 19$ \\
& $(0.95-0.74)$ & $(0.68-0.53)$ \\
\hline
\end{tabular}

Table 3 Stiffness of Package (false twist and spindle position)

\begin{tabular}{lcc} 
& Large false twist & Ordinary false twist \\
\hline Front row & 430 & 404 \\
Back row & 436 & 425 \\
\hline
\end{tabular}

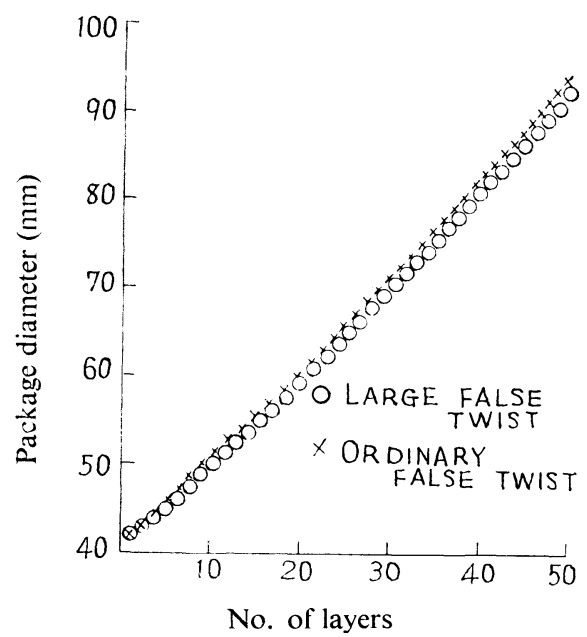

(a) Front row

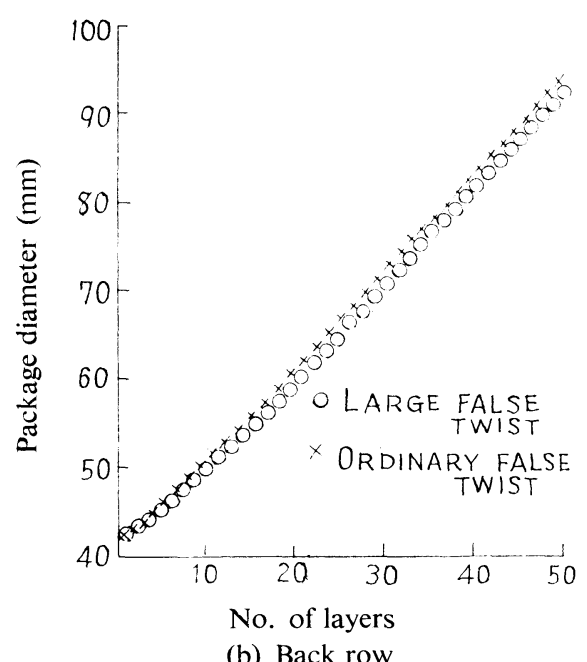

Fig.7 Package diameter (false twist)

The experimental data given in Figs. 7-9 and Tables 2-3 show, as regards the effect of false twists, that a roving given more false twists in some way or other tends to decrease in length and increase in tension (Fig. 8). Such a roving should increase winding tension as a matter of course, and the roving package will necessarily be wound more tightly (Table 3) and decrease slightly in diameter (Fig. 7). As the more twisted roving has more resistance to be stretched, the rate of draft during winding becomes smaller and the roving weight becomes larger (Fig. 9). 


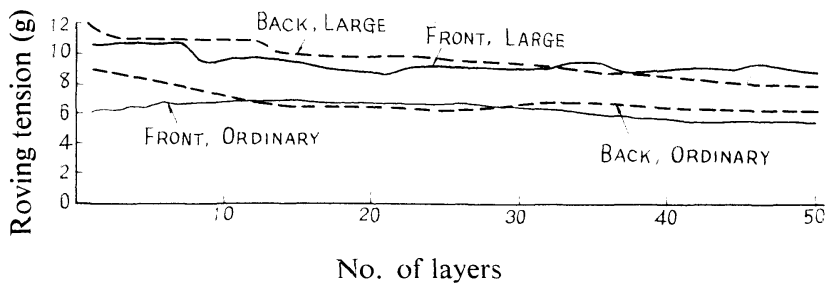

Fig.8 Roving tension (false twist, spindle position)

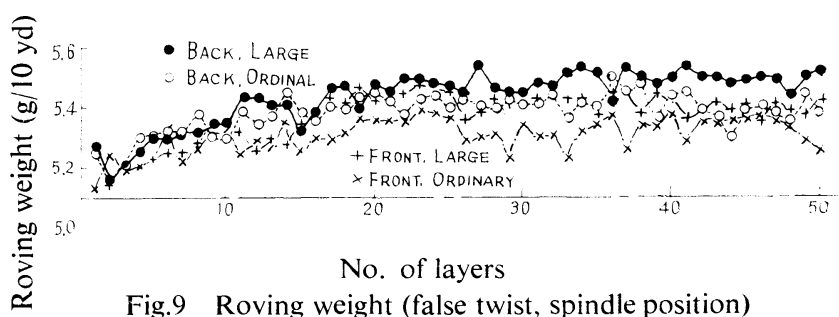

Fig.9 Roving weight (false twist, spindle position)

\section{Traverse Motion of Bobbin Rail}

\section{4-1. Experimental Procedures}

The apparatus used in this experiment measured and recorded the relative revolutions of the bobbin and the flyer and the diameter of the roving package continuously throughout winding. Therefore, fluctuations in these factors in layers were easily and minutely detected. Think of the weight of a yard of roving as roving weight. The angle displacement of the bobbin caused only by the traverse motion of the bobbin rail was recorded on paper wound on the bobbin by removing the motor pulley and driving the bobbin rail up and down manually. Additional revolutions of the bobbin caused by the traverse motion were calculable by the above displacement.

\section{4-2. Results of Expriment Discussed}

Typical examples of records of the relative revolutions of the bobbin and the flyer, the diameter of the roving package and roving tension are given in Figs. 10, 11 and 12. The experimental results concerning roving weight and improper revolutions of the bobbin due to the traverse motion are shown in Figs. 13 and 14, the additional angular velocity being obtained by differentiating geometrically the curve of the angular displacement.

Now a theoretical study of the winding of roving in a layer is made as follows. The volume of a roving delivered from the front roll per unit time $U_{\mathrm{F}}$ is expressible thus:

$$
U_{\mathrm{F}}=A_{\mathrm{F}} V
$$

$A_{\mathrm{F}}$ and $V$ denote respectively the sectional area of the roving and the delivery speed of roving from the front roll.

The volume of a roving fed to the flyer top per unit time $U_{\mathrm{gn}}$ is expressible as follows:

$$
U_{\mathrm{gn}}=W_{\mathrm{n}} A_{\mathrm{gn}}
$$

where $W_{\mathrm{n}}$ is the feeding speed of roving to the flyer top; and $A_{\mathrm{gn}}$, the sectional area of the roving converted so as to have the same density as that at the front roll. The suffix $\mathrm{n}$ denotes the number of layers in the roving package.

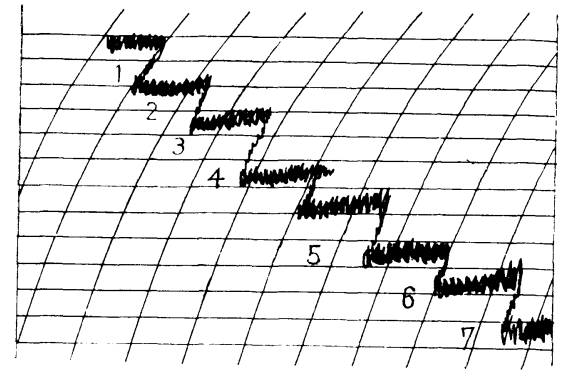

No. of layers

(a) Layers small in No.

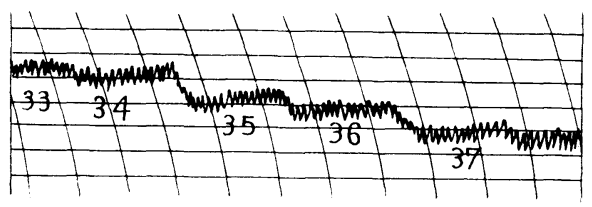

No. of layers

(b) Layers large in No.

Fig.10 Record of relative revolutions (traverse motion)

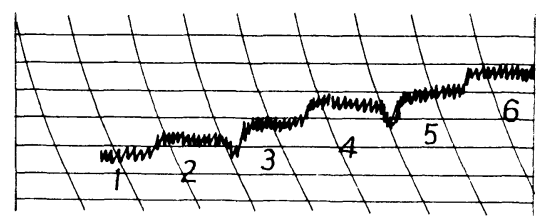

No. of layers

(a) Layers small in No.

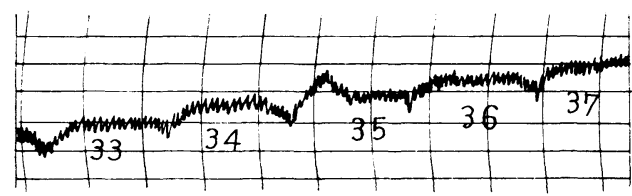

No. of layers

(b) Layers large in No.

Fig.11 Record of package diameter (traverse motion)

Assuming that a roving does not sag bewteen the front roll and the flyer top, and is extended only between them, then $W_{\mathrm{n}}$ should be

$$
W_{\mathrm{n}}=2 \pi r_{\mathrm{n}-.1} N_{\mathrm{n}}
$$

in which $r_{\mathrm{n}-1}$ is the diameter of the roving package and $N_{\mathrm{n}}$ is the number of relative revolutions of the bobbin and the flyer.

The volume of the roving between the front roll and the flyer top $V$ is expressible as

$$
V=l_{\mathrm{o}} A_{\mathrm{gu}}
$$

where $l_{\mathrm{o}}$ is the distance from the front roll to the flyer top.

To simplify the solution, assume that the winding draft rate is the same everwhere between the front roller and the flyer top. Variation of $V$ is, then, determined by the following equation, in the light of eqs. (1) and (2): 


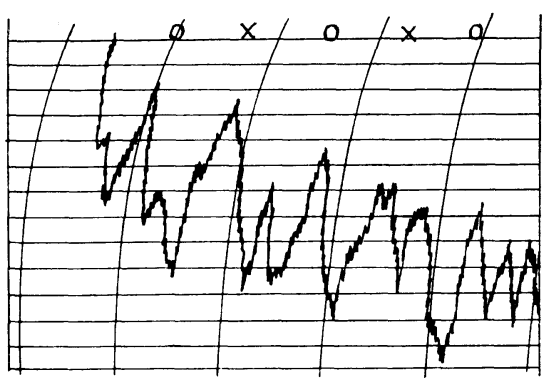

No. of layers

(a) Layers small in No.

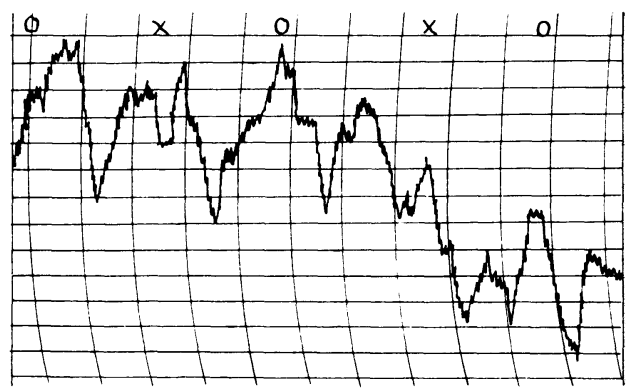

No. of layers

(b) Layers large in No.

Fig.12 Record of roving tension (traverse motion)

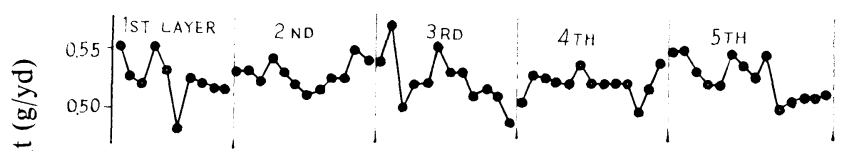

(a) Layers small in No.

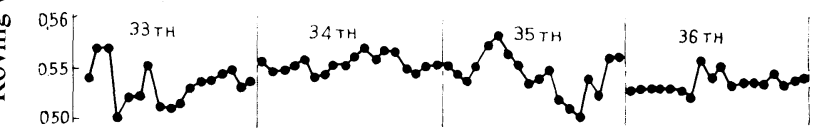

(b) Layers large in No.

Fig.13 Roving weight (traverse motion)

$$
\frac{d V}{d t}=U_{\mathrm{F}}-W_{\mathrm{n}} A_{\mathrm{gn}} \ldots \ldots \ldots \ldots \ldots \ldots \ldots \ldots
$$

Subsituting eq. (4) into eq. (5), we get eq. (6).

$$
\frac{d A_{\mathrm{gn}}}{d t}+\frac{W_{\mathrm{n}}}{l_{\mathrm{o}}} A_{\mathrm{gn}}=\frac{U_{\mathrm{F}}}{l_{\mathrm{o}}}
$$

The general soluticn of eq. (6) for a case where both $W_{n}$ and $U_{\mathrm{F}}$ are a function of time is obtained as follows.

$$
\begin{array}{r}
A_{\mathrm{g} n}=\exp \left(-\frac{1}{l_{0}} \int W_{\mathrm{n}}(t) d t\right)\left[\int\left\{\frac{U_{\mathrm{F}}(t)}{l_{\mathrm{o}}} \exp \left(\frac{1}{l_{\mathrm{o}}} \int W_{\mathrm{n}}(t) d t\right)\right\}\right. \\
d t+C] \\
\ldots \ldots
\end{array}
$$

in which $C$ is an integral constant which should be determined by the initial condition.

If $W_{\mathrm{n}}$ does not vary in layers, then

$$
A_{\mathrm{gn}}=\frac{U_{\mathrm{F}}}{W_{\mathrm{n}}}+C^{\prime} \exp \left(-\frac{W_{\mathrm{n}}}{l_{\mathrm{o}}} t\right)
$$

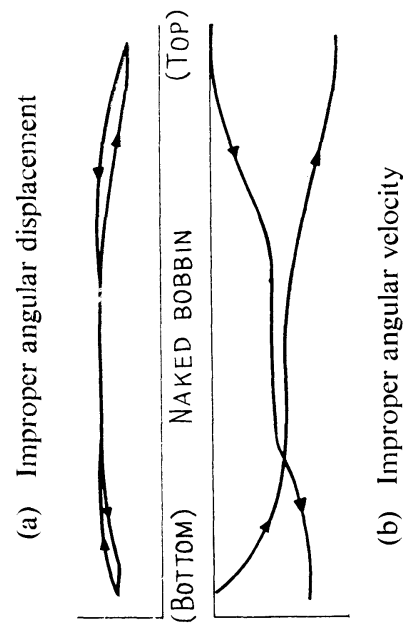

Fig.14 Tendency of improper displacement and velocity (traverse motion)

If this theoretical analysis is proper, this system has a self-operating control mechanism. Whatever the initial condition of the winding of a roving, $A_{\mathrm{gn}}$ approaches constant value $U_{\mathrm{F}} / W_{\mathrm{n}}$ with increase of time. The value $l_{\mathrm{o}} / W_{\mathrm{n}}$, the time which a roving takes to move from the front roll to the flyer top, is a time constant.

Roving weight being considered proportional to the sectional area of a roving, we can see fluctuations in them by the term of variation in $A_{\mathrm{gn}}$. The rate of winding draft is expressed as follows.

$$
D=A_{\mathrm{F}} / A_{\mathrm{gu}}
$$

Area $A_{\mathrm{F}}$ of a delivered roving is assumed to remain constant all the time. Then eqs. (7) and (8) with eq. (9) transform thus:

$\frac{1}{D}=\exp \left(-\frac{1}{l_{\mathrm{o}}} \int W_{\mathrm{n}}(t) d t\right)\left[\int\left\{\frac{V(t)}{l_{\mathrm{o}}} \exp \left(\frac{1}{l_{\mathrm{o}}} \int W_{\mathrm{n}}(t) d t\right) d t+C\right]\right.$

and

$$
\frac{1}{D}=\frac{V}{W_{\mathrm{n}}}+C^{\prime} \exp \left(-\frac{W_{\mathrm{n}}}{l_{\mathrm{o}}} t\right) \ldots \ldots \ldots \ldots\left(8^{\prime}\right)
$$

Fluctuation in relative revolutions shown in Fig. 10 agrees in their tendency with fluctuations assumed from the improper angular velocity in Fig.14. Fluctuations come in the growth of the package diameter in Fig.11 agree nealry with those assumed from the fluctuations in Fig.10. Fluctuations in the odd-number layers differ in tendency from fluctuations in the even-number layers. The differences are presumably caused by the difference in character between the curves of the improper angular velocity for the odd-number layers and those for the even-number layers in Fig.14.

What bearing do improper relative revolutions have on roving weight in various layers? Roving weight being considered proportional to $1 / D$, and $D$ proportional to the 
product of $r_{\mathrm{n}-1}$ by $N_{\mathrm{n}}$ in the light of eqs. (3), (8) and (9), variations in roving weight can be examined from the reciprocal of the product between the diameter of the package and relative revolutions. Fig. 15 shows this value obtained from Figs. 10 and 11, and seems to agree in the tendency of fluctuations with Fig.14, especially in the early stage of winding.

Fluctuations in the diameter of a package increase in the odd-number layers and decreases in the even-number layers (Fig.11). This tendency is noticeable at layers in the early stage of winding and presumably comes from the whirling motion of the bobbin and from change in the diameter of the package in a layer which is formed by fixing the initial position of winding nearly at a quater distance of the bobbin length from its bottom. Fluctuations in roving weight while layers are small in number (Fig.13(a)) are presumably influenced by these conditions. Eq.(7) assumes that the radius of the package is $r_{\mathrm{n}-1}$ for a certain region of layer and $r_{\mathrm{n}-1}+\delta$ for the succeeding region. This assumption includes a case where winding begins at one-quarter of a naked bobbin. $A_{\mathrm{gn}}$ for the radius at $r_{\mathrm{n}-1}$ is obtained as follows by eq.(8):

$$
A_{\text {gn }}=U_{\mathrm{F}} / W_{\mathrm{n}} \ldots \ldots \ldots \ldots \ldots \ldots \ldots \ldots \text { (10) }
$$

for steady state, assuming $U_{\mathrm{F}}(t)$ to be constant. Similarly, for the radius at $r_{\mathrm{n} . .1}+\delta$

$$
A_{\mathrm{gn}}=\frac{U_{\mathrm{F}}}{2 \pi N_{\mathrm{n}}\left(r_{\mathrm{n}}+\delta\right)}+C \exp \left\{-\frac{2 \pi N_{\mathrm{n}}}{l_{\mathrm{o}}}\left(r_{\mathrm{n}-1}+\delta\right) t\right\} \cdots
$$

If the initial condition of eq.(11) is taken as

$$
A_{\mathrm{gn}}=U_{\mathrm{F}} / W_{\mathrm{n}} \quad \text { at } t=0
$$

then Eq.(11) becomes as follows.

$A_{\mathrm{gn}}=\frac{U_{\mathrm{F}}}{W_{\mathrm{n}}\left(1+\frac{\delta}{r_{\mathrm{n}-1}}\right)}\left[1+\frac{\delta}{r_{\mathrm{n}-1}} \exp \left\{-\frac{W_{\mathrm{n}}}{l_{o}}\left(1+\frac{\delta}{r_{\mathrm{n}-1}}\right) t\right\}\right]$

The steady-state value of this equation is

$$
A_{\mathrm{gn}}=\frac{U_{\mathrm{F}}}{W_{\mathrm{n}}\left(1+\delta / r_{\mathrm{n} . .1}\right)}
$$

From eqs.(10) and (13) we may conclude that an abrupt change to the extent of $\delta$ in the diameter of the package gives $A_{\mathrm{g}}$ an influence in the form of $\delta / r_{\mathrm{n} . .1}$; and that, therefore, when $r_{\mathrm{n}-1}$ is still small, $\delta / r_{\mathrm{n}-1}$ is comparatively large and influences $A_{\mathrm{gn}}$ strongly. When $r_{\mathrm{n} .1}$ becomes large, $\delta / r_{\mathrm{n}-1}$ is small and its influence is limited. When $N_{\mathrm{n}}$ is changed in step shape to the extent of $s$ in a layer, $A_{\mathrm{gn}}$ is obtainable in a similar manner for steady state following a sudden change to the extent of $\varepsilon$ :

$$
A_{\mathrm{gn}}=\frac{U_{\mathrm{F}}}{W_{\mathrm{n}}\left(1+\varepsilon / N_{\mathrm{n}}\right)}
$$

A comparison of eqs. (13) and (13') shows that a variation in the radius of the package affects roving weight seriously while layers are small in number-or affects relative revolutions seriously if layers are large in number. The

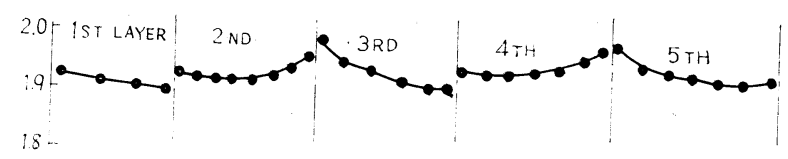

(a) Layers small in No.

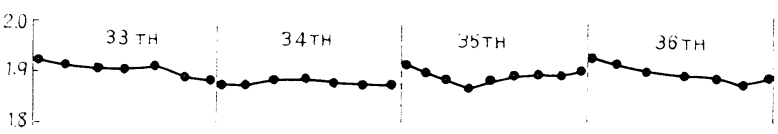

(b) Layers large in No.

Fig.15 Reciprocal of product of package diameter and relative revolutions of bobbin and flyer (traverse motion)

reason is that $\delta / r_{\mathrm{n}-1}$ becomes smaller with an increase in the number of layers, but that $s / N_{\mathrm{n}}$ cannot be decided to become small even if number of layers increase. These theoretical analysis agree roughly with the graphs plotted in Fig.13.

In addition to the above discussion a brief theoretical study of a case where a roving sags between the front roll and the flyer top is mentioned as follows. In this case, the length of a roving between the front roll and the flyer top should be considered a variable, assuming the sectional area of the roving to be constant. Then the volume of the roving between the front roll and the flyer top is expressible thus:

$$
V=A_{\mathrm{F}} l
$$

where $l$ is the length of the roving measured from the front roll to the flyer top. Eqs.(5) and (14) then lead to:

$$
l=\left(V-W_{\mathrm{n}}\right) t+C \ldots \ldots \ldots \ldots \ldots \ldots \ldots \ldots \ldots \ldots \ldots \ldots(15)
$$

The value $\left(V-W_{\mathrm{n}}\right)$ being usually controlled smaller than zero, $l$ decreases in any case. When $l$ equals $l_{0}$ the differential equation governing winding transforms into eq. (6). The fluctuations in roving tension shown in Fig. 12 seem to have some relation to the fluctuations in roving weight in Fig. 13. This relation, however, is not clear enough for any conclusions to be deducible from it.

\section{Relative Revolutions of Bobbin and Flyer}

\section{5-1 Experimental Procedures}

The relative revolutions of the bobbin and the flyer are variable by:

(1) Changing the shaper wheel;

(2) Using the belt shifter position regulator invented by one of the authors to adjust the winding conditions [9] or (3) Changing the initial position of the cone belt.

For certain reasons, we used method 3. To be more exact, we varied the relative revolutions of the bobbin and the flyer by fixing the initial belt position of the cone drum at a position corresponding to the 2nd and 4th layers in the usual winding. It was not possible to fix the initial position of the cone belt at a position corresponding to the 6th or a subsequent layer in the usual winding, because 
the roving would have sagged between the front roll and the flyer top.

5-2. Results of Experiment Discussed

The experimental results are shown in Figs. 16-19 and Table 4. "Ordinary," "2 layers later" and "4 layers later" refer to the usual winding and fixing the initial belt positions at positions corresponding to the 2 nd and the 4 th layers, respectively, in the usual winding.

The relative revolutions of " 4 layers later" were the smallest in number. Those in "Ordinary" were the largest in number (Fig.16). The following inference on the influence of relative revolutions is deducible:

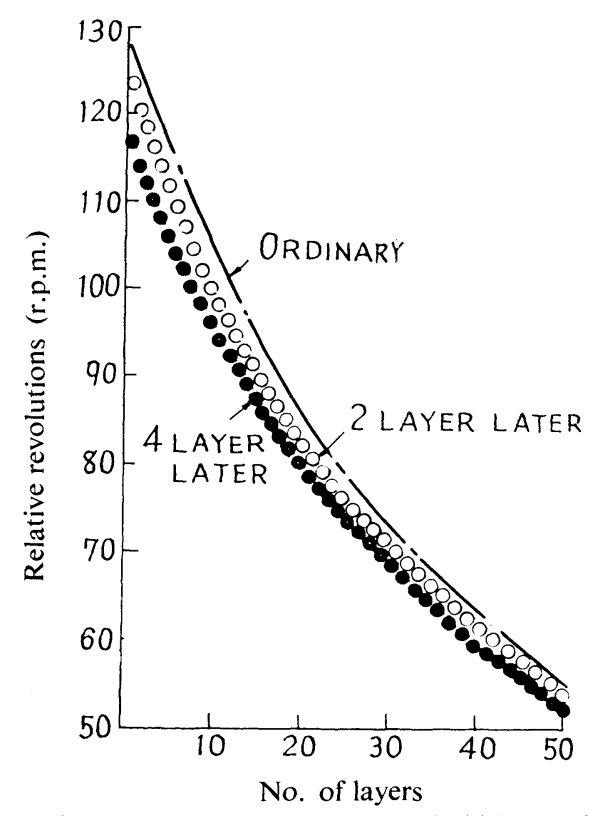

Fig.16 Relative revolutions of bobbin and flyer

Table 4 Stiffness of Package (relative revolutions)

$\begin{array}{crc}\text { Ordinary } & 2 \text { Layers Later } & 4 \text { Layers Later } \\ 390 & 380 & 325\end{array}$

The smaller the relative revolutions in number, the smaller the roving tension (Fig. 18), the smaller the winding draft and, therefore, the larger the roving weight (Fig. 19). The smaller the roving tension, the smaller the winding pressure of the presser. The smaller the winding pressure of presser, the larger the diameter of the package (Fig.17) and the softer the package (Table 4).

The fluctuations in some factors reveal interesting tendencies. Figs.17, 18 and 19 show that the gradient of the graph of the package diameter, the number of layers, the value of roving tension and the value of roving weight get close to some constant values as roving layers increase in number. This attests to the presence of a self-operating control mechanism of winding. This mechanism is clarified by the following theoretical analysis:

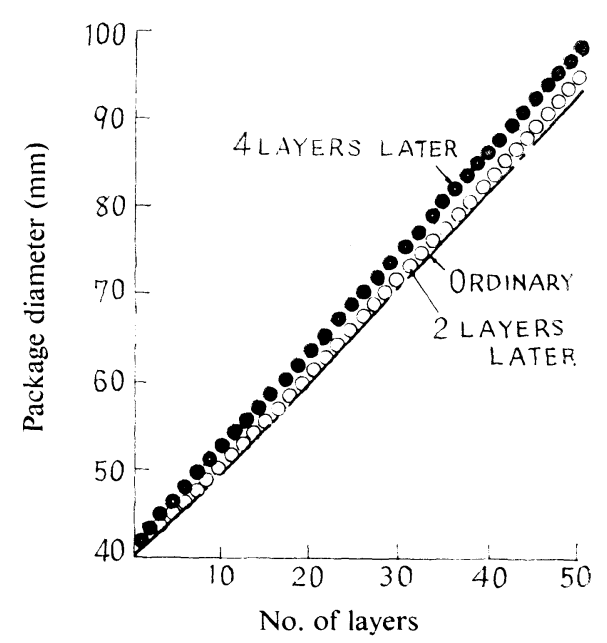

Fig.17 Package diameter (relative revolutions)

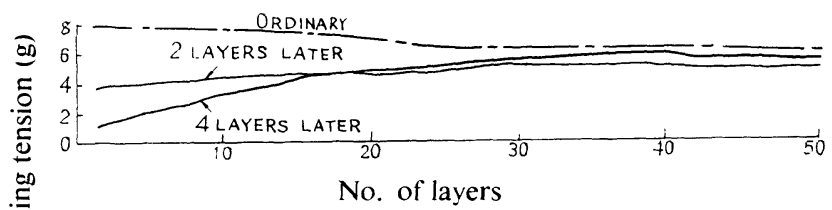

Fig.18 Roving tension (relative revolutions)

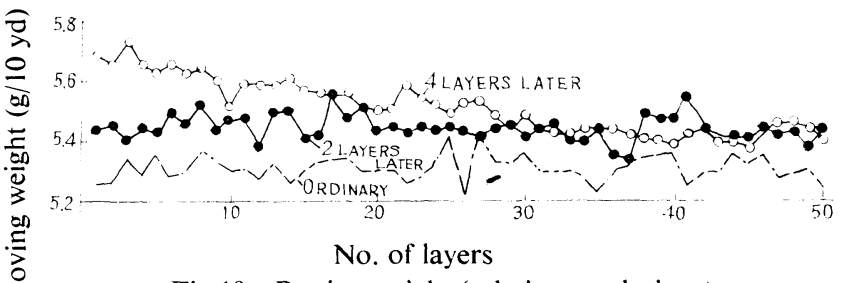

Fig.19 Roving weight (relative revolutions)

Assume that the shape of the cross section of a roving is rectangular, its width equal to pitch $b$ of winding in a layer and its height equal to increase $\Delta r_{\mathrm{n}}$ in the radius of a package per layer. The relation between $J r_{\mathrm{n}}$ and the the time required to wind around circumference $\Delta t_{\mathrm{n}}$, then, is :

$$
2 \approx r_{\mathrm{n}-1} b \Delta r_{\mathrm{n}}=\alpha A_{\mathrm{F}} V \Delta t_{\mathrm{n}}
$$

where $\alpha$ denotes an ajusting factor in presser pressure, etc. Ignoring variations in $r_{\mathrm{n}-1}, A_{\mathrm{F}}, V$, etc. in a layer, we obtain the relation between $\Delta r_{\mathrm{n}}$ and the time for winding layer $\Delta t^{\prime}$, namely:

$$
\Delta r_{\mathrm{n}}=\frac{\alpha A_{\mathrm{F}} V \Delta t_{\mathrm{n}}^{\prime}}{2 \pi r_{\mathrm{n}-1} b m_{\mathrm{n}}}
$$

$m_{\mathrm{n}}$ indicating the number of windings per layer.

$\Delta t^{\prime}{ }_{\mathrm{n}}$ being related to relative revolutions $N_{\mathrm{n}}$ by

$$
\Delta t^{\prime}{ }_{\mathrm{n}}=m_{\mathrm{n}} / N_{\mathrm{n}}
$$

eq.(17) transforms into:

$$
\Delta r_{\mathrm{n}}=\frac{\alpha A_{\mathrm{F}} V}{2 \approx r_{\mathrm{n}-1} b N_{\mathrm{n}}}
$$

Radius $r_{\mathrm{n}}$ of the roving package is expressed by the 
following equation:

$$
r_{\mathrm{n}}=r_{\mathrm{n} \cdot 1}+\Delta r_{\mathrm{n}}
$$

If $A_{\mathrm{F}}, V, b, N_{\mathrm{n}}$ and $r_{\mathrm{o}}$ (equal to the radius of naked bobbin $R$ ) are given then the values of $\Delta r_{1}, \Delta r_{2},-, J r_{\mathrm{n}}$ and $r_{1}, r_{2}, r_{3},-$, then $r_{n}$ is determined step by step by using eqs. (18) and (19) alternately. When $r_{n-1}$ is large, then $J r_{n}$ becomes smaller by eq.(18) and $r_{11}$ becomes smaller, too, by eq.(19).

If $r_{n-1}$ is small, then $\lrcorner r_{n}$ and, for that matter, $r_{\mathrm{n}}$ become large. This relation is a mathematical expression of the self-operating control of winding.

To know the influence of a set of relative revolutions $N_{1}, N_{2,-}, N_{11}$ to $\lrcorner r_{11}$ and $r_{11}$ at a glance without the trouble of numerical calculations, treat eq.(17) as follows: The number $n$ of layers in this equation takes only an integer as a matter of course. Therefore, $\lrcorner r_{n}, r_{n}, N_{n}$ and $\Delta t^{\prime}{ }_{n}$ also take only discrete values. However, $\Delta r_{n}$ being smaller than any $r_{11}$, we may be permitted, within the limits of a minor error, to treat $n$ and, for that matter, $\Delta r_{\mathrm{n}}, r_{\mathrm{n}}, N_{\mathrm{n}}$ and $\Delta t^{\prime}{ }_{\mathrm{n}}$ as if they were continuous quantities. Then increment of the time is expressible as follows:

$$
\Delta t_{\mathrm{n}}{ }^{\prime}=\frac{m_{n}}{N_{n}} J_{n}
$$

where $\Delta n$ means an increase in the number of layers, even though it is meaningless physically. Substituting eq.(20) into eq.(17) gives us:

$$
J r_{n}=\underset{J_{\mathrm{F}} V}{2-r_{n-1} b N_{n}}
$$

$\alpha$ is omitted from this equation, because our purpose here is only to see the self-operating control mechanism of winding. $N_{n}$ in eq. (21) is a quantity fixed before the beginning of winding.

Assume, for convenience's sake, that $N_{\mathrm{n}}$ is fixed to fit only a case where $r_{\mathrm{n}}=\bar{r}_{\mathrm{n}}, J r_{\mathrm{n}}=\bar{J} r_{\mathrm{n}}, A_{\mathrm{F}}=\overline{A_{\mathrm{F}}}$ and $V=\bar{V}$, and to satisfy the following equation:

$$
2 \pi \bar{r}_{\mathrm{n}-1} N_{\mathrm{n}} \overline{\mathrm{J}} r_{\mathrm{n}} b=\bar{A}_{\mathrm{F}} \bar{V}
$$

Eq.(21), then, transforms into:

$$
\frac{\Delta r_{\mathrm{n}}}{\Delta n}=\overline{J r_{\mathrm{n}}} \frac{A_{\mathrm{F}}}{A_{\mathrm{F}}} \cdot \frac{V}{\bar{V}} \cdot \frac{\overline{r_{\mathrm{n}-1}}}{r_{\mathrm{n}-1}}
$$

Taking limit of the above equation for $\lrcorner n \rightarrow 0$ we get

$$
\left.\frac{d r}{d n}=\right\lrcorner r \frac{A_{\mathrm{F}}}{A_{\mathrm{F}}} \cdot \frac{V}{V} \cdot \frac{r}{r}
$$

where $\frac{d r}{d n}$ means the limit of the quantity $\frac{d r_{n}}{\Delta n}$ for $J n \rightarrow 0$. Quantity $\frac{d r}{d n}$ may be considered an indicator of the sectional area of a roving and, accordingly, of the roving weight. Eq.(22), integrated, transforms into:

$$
r^{2}=2 \int \overline{\Delta r} \frac{A_{\mathrm{F}}}{A_{\mathrm{F}}} \frac{V}{\bar{V}} r d n+C
$$

where $C$ is an integral constant. Assuming that $V, \bar{V}, A_{F}$ and $\overline{A_{\mathrm{F}}}$ are not a function of $n$, then eq.(23) is soluble as follows:

$$
\frac{r^{2}}{\overline{\mu^{*}}}-k^{2}\left(n+\frac{R}{\overline{4}}\right)^{2}=C^{\prime} \text { for } r \geq R, n \geqq 0 \quad \cdots
$$

where $C^{\prime}$ is integral constant and

$$
k \equiv \sqrt{\frac{A_{\mathrm{F}}}{\overline{A_{\mathrm{F}}}} \frac{V}{V}}
$$

Eq.(24) being a hyperbola, and $C^{\prime}$ should be fixed by the initial condition of winding. To simplify explanation, assume a case where $A_{\mathrm{F}}=\overline{A_{\mathrm{F}}}, V=\bar{V}$ and so $k=1$. Eq.(24), then, transforms into:

$$
\frac{r^{*}}{\sqrt{r^{2}}}-\left(n+\frac{R}{\sqrt{r}}\right)^{:}=C^{\prime} \text { for } r \geqq R, n \geqq 0
$$

The initial condition being usually

$$
r=R \quad \text { at } n=0
$$

eq.(25) transforms into:

$$
r=R+\overline{\Delta v}
$$

which denotes proper winding.

Note that eq.(26) is really the asymptote of hyperbola eq.(25) and, that, therefore, whatever the initial condition of winding may be, $r$ governed by eq.(25) approaches, by degrees, the value of $r$ governed by eq.(26) with an increase in variable $n$. In case of " $n_{\text {o }}$ layers later," the initial condition is

$$
r=R \quad \text { at } n=n_{\text {o }}
$$

Eq.(25), therefore, transforms into:

$$
\left(n+\frac{R}{\lrcorner r}\right)^{2}-\frac{r^{2}}{\lrcorner r^{2}}=n_{0}, \cdots\left(1+\frac{2 R}{J r n_{0}}\right) \text { for } r \geq R, n \geq n_{0} \cdots
$$

In this equation $n$ denotes the belt position of the cone drum if we take the initial condition as $r=R$ at $n=n_{\mathrm{o}}$. To compare this theory with the experimental results, substitute $n+n_{0}$ for $n$. This leads to an equation on layer parameter $n$ :

$$
\left(n+\frac{R}{\bar{J}}+n_{0}\right) 2-\frac{r^{2}}{\sqrt{r^{2}}}=n_{0}^{2}\left(1+\frac{2 R}{\sqrt{r} n_{0}}\right) \text { for } r \geqq R, n \geqq 0(28)
$$

the asymptote of which is

$$
r=\overline{\Delta r}\left(n+n_{\mathrm{o}}\right)+R
$$

The fluctuations in the diameter of the roving package for the number of layers cited in Fig. 17 agree in their relative position of the curve with those obtained by eq.(28). However, the various curves in Fig.17 do not match in shape those obtained by eq.(28). For example, the curve for "Ordinary" in Fig. 17 is a downward convex, while the curve obtained by eq.(28) is a straight line. This disagreement between experiment and theory seems due to the ignoring of the influence of presser pressure and to the assumption of $A_{\mathrm{F}}=\overline{A_{\mathrm{F}}}$ and $V=\bar{V}$ in the theory. Only for the influence of the latter condition the following analysis may be led safely. 
When $k$ is less than 1, e.g., $A_{\mathrm{F}}<\overline{A_{\mathrm{F}}}$ and $V=\bar{V}$ or $A_{\mathrm{F}}=\overline{A_{\mathrm{F}}}$ and $V=\bar{V}$, the curve of $r: n$ given by eq.(24) is not a straight line but a downward convex like that of "Ordinary" in Fig. 17.

To see the fluctuations in roving weight, obtain $d r$ ! $d n$ from eq.(28) as an indicator of roving weight.

$$
\frac{d r}{d n}=\frac{\overline{\Delta r}}{\sqrt{1-\frac{n_{0}^{2}\left(1+\frac{2 R}{\Delta r} n_{0}\right.}{\left(n+\frac{R}{\Delta r}+n_{0}\right)^{2}}}} \quad \ldots \ldots \ldots \ldots \ldots \ldots \ldots
$$

This equation denotes that the value $d r / d n$ becomes $\bar{J}$ for $n \rightarrow \infty$. For a more general case, we get from Eq.(24)

$$
\frac{d r}{d n}=\frac{k^{\prime \prime}\left(\overline{J r}+\frac{R}{n}\right)}{\sqrt{\frac{C^{\prime}}{n^{2}}+k^{2}\left(1+\frac{R}{\Delta r n}\right)^{2}}}
$$

and

$$
d r / d n \rightarrow \overline{k\lrcorner r} \quad \text { for } n \rightarrow \infty
$$

Eqs.(30)-(32) show the self-operating control of roving weight. Fluctuations in roving weight for the number of layers in Fig.19 agree on the whole in their tendency with the curve of $\frac{d r}{d n}: n$ given by eqs.(30)-(32), although there is a noticeable discrepancy between theory and experiment, presumably due to ignoring presser pressure in the theory.

It seems safe to say that, if the relative revolutions of the bobbin and the flyer is properly fixed for a given roving fed to a frame in a given condition, the values affecting the state of winding-package diameter, roving tension, and roving weight, etc. - will gradually become proper-with a certain limitation-as layers increase in number, no matter what roving is fed and no matter what the initial condition of winding is.

\section{Spindle Revolutions}

\section{6-1. Experimental Procedures}

The spindle revolutions were fixed at 546, 820 and 945 r.p.m., the motor pulley being varied in diameter to vary the number of spindle revolutions. As we said in Part 1, presser pressure plays an important role in the winding of roving and is influenced considerably by the number of spindle revolutions. To develop a high-speed winding frame, we should design the presser of the flyer to keep it from exerting heavy pressure on the package. The heavier the presser pressure, the larger the roving tension and the more chances of roving breakage. To make winding possible with the various numbers of revolutions and to keep the effects of presser pressure as uniform as possible in each instance, we remade pressers by the method and with the equipment described in [2],[10].

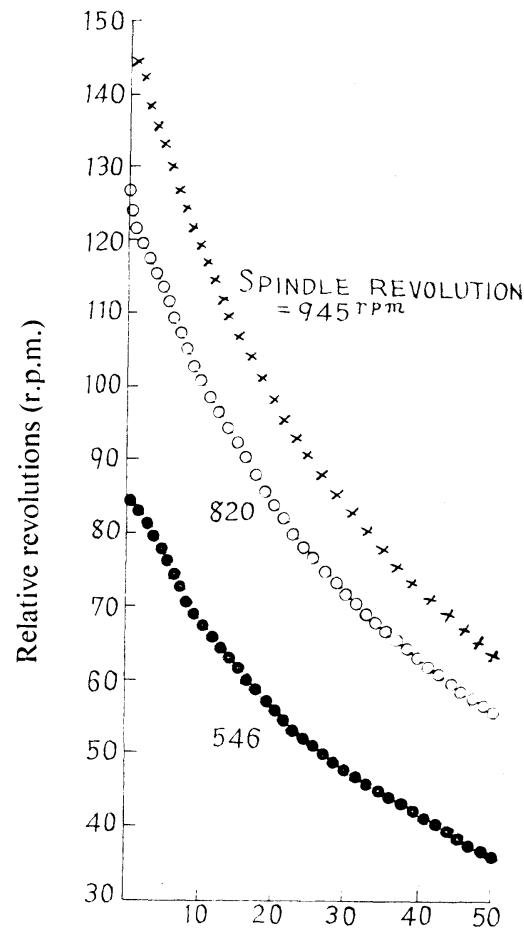

Fig.20 Relative revolutions of bobbin and flyer (spindle revolutions)

\section{6-2 Results of Experiment Discussed}

The experimental results are shown in Figs.20-23 and Table 5. They show that the diameter of the roving package decreases (Fig.21), package stiffness increases (Table 5), roving tension decreases (Fig.22) and roving weight increases (Fig.23) with an increase in the number of spindle revolutions.

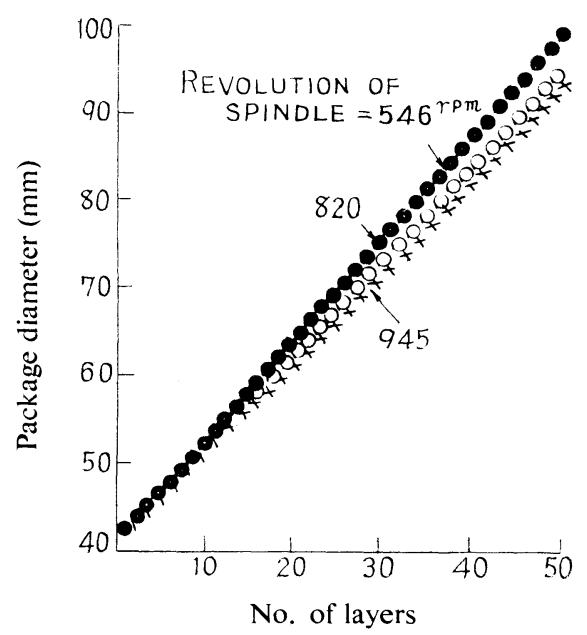

Fig.21 Package diameter (spindle revolutions) 


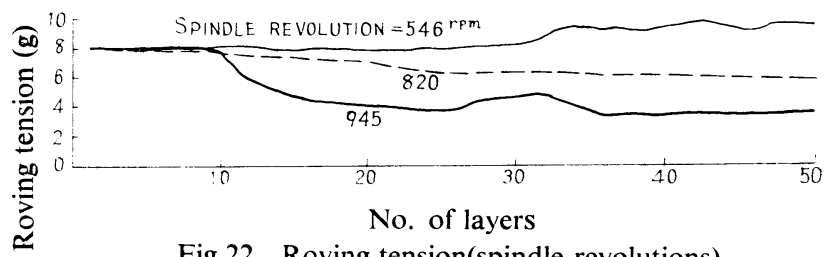

Fig.22 Roving tension(spindle revolutions)

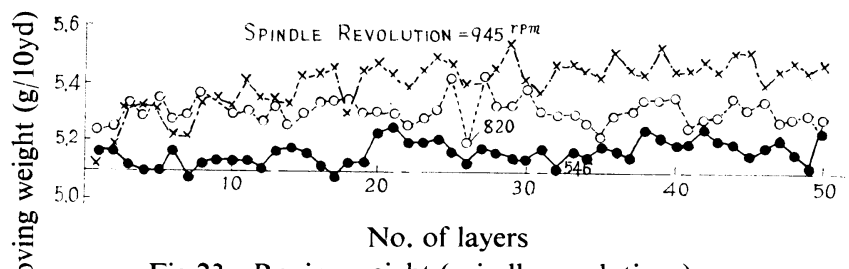

Fig.23 Roving weight (spindle revolutions)

Table 5 Stiffness of Package (spindle revolutions)

\begin{tabular}{ll}
\hline Spindle revolutions & \\
\hline 546 r.p.m. & 346 \\
820 & 390 \\
945 & 460 \\
\hline
\end{tabular}

With spindle revolutions fixed at a large number, one would think that the effects of such factors as whirling of the bobbin and the spindle, mechanical vibrations of the frame and inertia forces of the roving (these need not worry us in winding at a low speed) show up conspicuously and that there must be adjustment error of remade pressers. On the whole, these factors and the adjustment error seemed to increase winding pressure in our experiment, because the experimental results close resembled in tendency the results for higher presser pressure.

Presumably, then, winding pressure and, therefore, presser pressure are among the most important factors in making proper winding possible on a high-speed frame.

\section{Conclusions}

It seems safe to conclude from our experiments and theoretical analysis of their results that:

1) Roving weight seems to be influenced very much by the diameter of the roving package, the diameter being influenced very much by the winding pressure of the presser. This relation holds true in all cases.

2) The winding mechanism of the roving frame has some kind of self-operating control mechanism, which has no doubt been endowed by the long experience of engineers without their being aware of it. To develop a better winding mechanism, we have to devise one having self controlling mechanism with our eyes wide open.

\section{Literature cited}

[1] T. Isshi; J. Text. Mach. Soc. Japan, English ed., Vol.8, No.2, p. 15 (1962)

[2] T. Isshi; J. Text. Mach. Soc. Japan, Japanese ed., Vol. 15, p. 169 (1962)

[3] K. Fujino, et al; Textile Machinery, Japan Soc. of Mech. Engs. (1958)

[4] Report from Technical Committee on Preparatory Spinning (II); J. Text. Mach. Soc. Japan, Japanese ed., Vol. 13, p. 48 (1960)

[5] F. Schmoller; Melliand. Textilber., Vol. 35, p. 611 (1954)

[6] T. Takasugi; Boshoku-kai, vol. 39, p. 379 (1950)

[7] S. Noda, et al.;J. Text. Mach. Soc. Japan, Japanese ed., Vol. 3, p. 332 (1950)

[8] Y. Miura; J. Text. Mach. Soc. Japan, Japanese ed., Vol. 6, p. 422 (1953)

[9] S. Watanabe, et al.; Report of Japan Cott. Tech. Inst., No. 27 (1958)

[10] T. Isshi; J. Text. Mach. Soc. Japan, Japanese ed., Vol. 14, p. 911 (1961) 